Artículo de reflexión

\title{
Michel Foucault: escuela, sexualidad y procesos de subjetivación ${ }^{1}$
}

\author{
Michel Foucault: school, sexuality and subjective \\ processes
}

\author{
Katherine Traslaviña Castillo ${ }^{2}$ \\ Universidad Nacional Autónoma de México \\ Gabriel Macías $\mathrm{Cruz}^{3}$ \\ Universidad Nacional Autónoma de México
}

Recepción: 18 de julio del 2021

Evaluación: 24 de agosto del 2021

Aceptación: 26 de agosto del 2021

\footnotetext{
1 El presente artículo fue escrito en el marco del trabajo de investigación "Educación sexual y biopolítica: análisis de los procesos de subjetivación desde el pensamiento de Michel Foucault", desarrollado en el Programa de Posgrado de Pedagogía de la UNAM, México.

2 Maestra en pedagogía por la Universidad Nacional Autónoma de México, Licenciada en Pedagogía Infantil por la Universidad Distrital Francisco José de Caldas de Colombia.

Correo electrónico: katha_castillo@hotmail.com

3 Candidato a Doctor en Pedagogía de la Universidad Nacional Autónoma de México.

Correo electrónico: didactiel@hotmail.com
} 


\title{
Resumen
}

De acuerdo con Foucault, los procesos de subjetivación se enmarcan en formaciones históricas. A cada formación histórica corresponden verdades catalogadas como legítimas y tecnologías de poder, que en conjunción son capaces de dirigir conductas y producir determinado tipo de sujeto. Actualmente la escuela es una institución en la que convergen tecnologías de poder (como la disciplina y la biopolítica) y saberes concebidos como verdaderos (por ejemplo, la sexualidad), que propician procesos de subjetivación. La disciplina tendrá como blanco de poder al cuerpo en tanto individuo, y la biopolítica se encargará del conjunto de cuerpos: la población. En este artículo analizaremos las formas en las que la escuela, como institución disciplinaria y de control, orienta las prácticas de los individuos mediante el saber de la sexualidad, para que devengan en sujetos sexualizados capaces de autorregularse, con la finalidad de defender un conjunto: la sociedad.

Palabras clave: escuela, disciplina, biopolítica, sexualidad, procesos de subjetivación.

\begin{abstract}
According to Foucault, the processes of subjectivation are framed in historical formations. Each historical formation corresponds to truths classified as legitimate and power technologies, which, in conjunction, are capable of directing behaviors and, thus, producing a certain type of subject. Currently, the school is situated as an institution within which power technologies converge (such as discipline and biopolitics) and knowledge conceived as true (for example, sexuality), to, from them, promote subjectivation processes. The discipline will have as a target of power the body as an individual and, biopolitics will be in charge of the set of bodies: the population. In this way, in this article of reflection, we will analyze the ways in which the school, as a disciplinary and control institution, guides the practices of individuals, through the knowledge of sexuality, so that they become sexualized subjects capable of self-regulation, in order to defend a group: society.
\end{abstract}

Keywords: school, discipline, biopolitics, sexuality, subjectivation processes. 


\section{Introducción}

Michel Foucault ha sido reconocido por su amigo, el historiador Paul Veyne, como quien revolucionó la historia (1984, p. 199). Ello por la forma de proceder del filósofo francés en sus investigaciones, al asumir la historia como formaciones históricas con discontinuidades y singularidades, que con sus respectivos discursos y prácticas hacen posible la formación de sujetos. Es por esto que en su trabajo filosófico se podrán advertir tres ejes principales: el saber, el poder y la subjetivación. Es decir, para Foucault, dentro de cada formación histórica nos constituimos como sujetos de conocimiento, sujetos capaces de influir sobre el comportamiento de los otros mediante el ejercicio de poder, y como sujetos éticos. De esta forma, el filósofo francés se interesa por responder a la pregunta de cómo hemos llegado a ser lo que somos actualmente, esto es, a plantear una ontología del presente, "(...) un análisis de la constitución histórica de nuestra subjetividad (...)" (Castro, 2011, p. 285). En consecuencia, los procesos de subjetivación responden a lo que en cada época se considera como verdad. En tal sentido, un sujeto es el resultado de un proceso histórico. Los procesos de subjetivación responden a formaciones históricas.

De acuerdo con lo anterior, en el presente artículo buscamos reflexionar sobre las formas en las cuales la escuela, como institución disciplinaria y de control, opera en la producción de subjetividades, tomando como ejemplo el saber de la sexualidad. Para esto es pertinente indagar sobre las tecnologías de poder que intervienen: la anatomopolítica del detalle y la biopolítica. La primera tiene como blanco de poder el cuerpo-individuo, y se centra en el ejercicio de la disciplina para, desde el más mínimo detalle, intervenir en el encauzamiento de determinada conducta. La segunda, la biopolítica, se ocupa del cuerpo en tanto especie, es decir, gestiona, regula y administra un conjunto de personas, configurando así formas de vida deseables para el bienestar de la sociedad. En suma, se trata de analizar las formas de operación de las tecnologías de poder dentro de la escuela, bajo el discurso de la sexualidad, en la configuración de determinados procesos de subjetivación: el sujeto sexualizado.

\section{Tecnologías de poder: anatomopolítica y biopolítica}

La anatomía política del detalle o anatomopolítica es equivalente, en palabras de Foucault, a una “(...) mecánica del poder, [la cual] define cómo se puede 
hacer presa en el cuerpo de los demás, no simplemente para que ellos hagan lo que se desea, sino para que operen como se quiere, con las técnicas, según la rapidez y la eficacia que se determina (...)" (2009, p. 141). Su ejercicio de poder estará determinado por la disciplina.

En el pensamiento de Foucault se pueden distinguir dos formas de comprender la disciplina. Una articulada al orden del saber y, la otra, al orden del poder. La disciplina, en el orden del saber, se comprende como una formación discursiva que tiene por objetivo la producción y regulación de los discursos. En este sentido, Foucault planteará cómo, desde finales del siglo XVIII, el Estado ha intervenido en la disciplinarización y ordenamiento de los discursos a partir de la descalificación y eliminación de los saberes que se consideran inútiles, así como en la jerarquización mediante la clasificación, desde los más particulares a los generales y, en la normalización de estos. Es en este marco de organización en el que surge la ciencia, ya no las ciencias. Al respecto, el filósofo francés plantea:

El siglo XVIII fue el siglo del disciplinamiento de los saberes, es decir, la organización interna de cada uno de ellos como una disciplina que tiene, en su campo de pertenencia, a la vez criterios de selección que permiten desechar el falso saber, el no saber, formas de normalización y de homogeneización de los contenidos, formas de jerarquización y, por último, una organización interna de centralización de esos saberes en torno de una especie de axiomatización de hecho. Por lo tanto, ordenamiento de cada saber como disciplina y, por otra parte, exposición de esos saberes así disciplinados desde adentro, su puesta en comunicación, su distribución, su jerarquización recíproca en una suerte de campo o disciplina global que se denomina, precisamente, la ciencia. Antes del siglo XVIII, la ciencia no existía. Había ciencias, había saberes y también estaba, si ustedes quieren, la filosofía (2000, p. 170).

Un ejemplo de este sistema de organización lo podemos encontrar en la universidad y en la escuela, a partir de la clasificación, selección, jerarquización e institucionalización de ciertos saberes, lo que conlleva a la eliminación de otros. En consecuencia, una forma de enseñanza centrada en las formas de enunciación, ya no en el contenido mismo, es la que impera.

Por otro lado, la disciplina en el orden del poder remite a las tecnologías de poder que buscan una individualización disciplinada del sujeto. El cuerpo se convierte, entonces, en el objeto de poder, como forma de organización interna 
en lugares específicos y sus movimientos en términos de eficacia. El poder disciplinario se comprende como una vía de encauzamiento del individuo, de enderezamiento de su conducta, para producirlo y transformarlo: "El cuerpo humano entra en un mecanismo de poder que lo explora, desarticula y recompone" (2009, p. 160). De esta forma, la disciplina captura los cuerpos, captura el tiempo y la vida del individuo, establece métodos meticulosos de dirección de las actividades y operaciones del cuerpo, garantizando así la sujeción de sus fuerzas para hacerlas útiles, en términos político-económicos, y dóciles en términos éticos. Esto significa que la fuerza del cuerpo debe estar encaminada a su productividad económica, y la docilidad debe seguir un conducto de valores normalizados dentro de la sociedad.

En este sentido, es importante señalar que en la disciplina no se trata de represión, esclavitud o servidumbre, pues su fin no es la dominación por sí misma. La disciplina no es una relación opresor-oprimido, una apropiación de los cuerpos, ni una dominación inquebrantable sin análisis, sino la constitución de relaciones de poder estructuradas por determinados saberes. Por ejemplo, un joven que se masturba demasiado puede acarrear problemas en términos de productividad; ya no será útil, pues no tendrá la misma fuerza que aquel que no lo hace. A su vez, el joven que se masturba sabe que no debe hacerlo en espacios públicos (la escuela, el trabajo, frente a sus padres) porque esto sería concebido como una mala conducta, una falta moral, sería considerado un sin vergüenza, un indócil. De acuerdo con esto, una microfísica del poder se gesta mediante una anatomía política de detalle:

La disciplina aumenta las fuerzas del cuerpo (en términos económicos de utilidad) y disminuye esas mismas fuerzas (en términos políticos de obediencia). En una palabra: disocia el poder del cuerpo; de una parte, hace de este poder una "aptitud", una "capacidad" que trata de aumentar, y cambia por otra parte la energía, la potencia que de ello podría resultar, y la convierte en una relación de sujeción estricta. Si la explotación económica separa la fuerza y el producto del trabajo, digamos que la coerción disciplinaria establece en el cuerpo el vínculo de coacción entre una aptitud aumentada y una dominación acrecentada (p. 160).

El individuo disciplinado responde a cuatro características: 1) la distribución de los cuerpos en el espacio, para lo cual se clausuran ciertos lugares con respecto a otros, por ejemplo, el colegio, la fábrica o los cuarteles. De la misma manera hay división de zonas y cada individuo ocupa un lugar específico, en 
el que la disciplina organiza un espacio en términos de utilidad; 2) sumado a esto está el control de la actividad, en el que el empleo del tiempo debe ser completamente útil. En consecuencia, es necesario que el individuo realice pronto su actividad y se prohíban los momentos de ociosidad; 3) la tercera característica refiere a la organización de las creaciones o producciones. Ésta se encarga de dividir la totalidad del proceso en segmentos temporales que vayan de lo simple a lo complejo. Esto se lleva a cabo en términos de un tiempo evolutivo, como por ejemplo los planes de estudio en los colegios; 4) por último, la composición de las fuerzas, las aptitudes y capacidades individuales se evidencian en una colectividad útil. Esto significa "Reducción funcional del cuerpo. Pero también, inserción de este cuerpo-segmento en todo un conjunto sobre el cual se articula" (p. 191).

En consecuencia, existen medios o instrumentos del buen encauzamiento para la individualización disciplinada: la vigilancia jerárquica, la sanción normalizadora y el examen. La primera consiste en una práctica donde reina el sentirse observado, examinado y controlado aun si el vigilante no está presente. La segunda se plantea en términos correctivos, estableciendo lo permitido y lo prohibido, instaurando un principio de coerción a partir de pequeños mecanismos penales que deben evitar las desviaciones. El tercero examina, clasifica, califica y corrige, haciendo de cada individuo un caso, un objeto de conocimiento visible basado en un saber especifico (pedagogía, medicina, psiquiatría, etc.), en el que el examen se establece como el núcleo de los procedimientos que constituyen al individuo como objeto y efecto del saber y el poder. Mediante el examen se establece, entonces, un sistema de registro del individuo, un campo documental acerca de él, que constituye la formación de códigos de individualidad disciplinaria. Existe pues “(...) una maquinaria de control que ha funcionado como un microscopio de la conducta (...)" (p. 203).

La segunda tecnología de poder será la biopolítica. Surge en la modernidad, se anuda a un saber médico y tiene por objetivo el control de la vida biológica mediante el poder político, es decir, que a partir de mecanismos de poder se busca la regulación de la población. Para ello se tiene en cuenta el cuerpo en su conjunto, no de forma individualizada como sucedía con la disciplina, ya que según Foucault la biopolítica “(...) está destinada a la multiplicidad de los hombres, pero no en cuanto se resumen en cuerpos sino en la medida en que forman, al contrario, una masa global, afectadas por procesos de conjunto 
que son propios de la vida, como el nacimiento, la muerte, la producción, la enfermedad, etcétera (...)” (2000, p. 220). Así, la biopolítica no sugiere una superación de la disciplina; por el contrario, existe una correlación y articulación entre ambas a fin de conducir la conducta de los individuos y producir sujetos.

En el pensamiento del filósofo francés no hay una teoría general o noción única de biopolítica. A lo largo de sus trabajos se evidencian, más bien, cuatro enfoques que, si bien no son antagónicos, presentan determinados rasgos que a continuación serán descritos. El primer enfoque lo encontramos en la conferencia Nacimiento de la medicina social, en la que la biopolítica está atravesada por el surgimiento de la medicina social. La segunda perspectiva se encuentra en el apartado "Derecho de muerte y poder sobre la vida" de su libro Historia de la Sexualidad, Voluntad de saber, en la que esta tecnología se establece como un poder de "hacer vivir o dejar morir", diferenciándose del poder soberano. En este apartado, la biopolítica estará analizada en el marco de la reflexión sobre el arte de gobernar un fenómeno social de reciente surgimiento: la población. Hallamos el tercer enfoque en su curso Defender la sociedad, en el que se aborda a la biopolítica en relación con la guerra de razas y, por último, en Seguridad, territorio y población, en donde se analiza la biopolítica en relación con la gubernamentalidad.

Foucault establece ciertos dispositivos de seguridad de la biopolítica que no pasan por la imposición de una ley o la prohibición, sino por ajustarse a unos límites aceptables. Es decir, se calculan los costos de reprimir o tolerar ciertas conductas, estableciendo una medida considerada como óptima, ya que el objetivo de los dispositivos de seguridad es la consolidación del Estado, no en términos de progreso o de una evolución del mismo hacia otra forma de gobierno, sino por su ser en sí mismo. En este sentido, el Estado requiere de otro arte de gobernar que no esté solamente sustentado por un marco jurídico, sino, principalmente, que se apoye en una gubernamentalidad basada en la libertad, una administración de las cosas que tenga presente la libertad de los hombres, es decir, a partir de mecanismos permisivos.

Así, la biopolítica se caracteriza por tener en cuenta un conjunto de procesos susceptibles de regular en relación con la vida política de los individuos, tales como el clima, el comercio, el entorno material, los valores morales. Tal como afirma Foucault: “(...) la biopolítica va a extraer su saber y definir 
el campo de intervención de su poder en la natalidad, la morbilidad, las diversas incapacidades biológicas, los efectos del medio" (p. 222), y a su vez, tendrá presente la circulación de hombres y cosas teniendo en cuenta el elemento de la libertad y, por último, la fijación de la norma a partir de curvas de normalidad. Ya que población y medio tienen una relación simbiótica, una relación viva, es necesario que el Estado vigile y controle esta relación, ejerza su poder sobre la población en tanto seres vivos con los procesos naturales que lo rodean, con el fin de establecer formas de vida saludables y productivas. En palabra de Foucault:

Será preciso modificar y bajar la morbilidad; habrá que alargar la vida; habrá que estimular la natalidad. Y se trata, sobre todo, de establecer mecanismos reguladores que, en esa población global con su campo aleatorio, puedan fijar un equilibrio, mantener un promedio, establecer una especie de homeostasis, asegurar compensaciones; en síntesis, de instalar mecanismos de seguridad alrededor de ese carácter aleatorio que es inherente a una población de seres vivos; optimizar, si ustedes quieren, un estado de vida: mecanismos, podrán advertirlo, como los disciplinarios, destinados en suma a maximizar fuerzas y a extraerlas, pero que recorren caminos enteramente diferentes (p. 223).

En suma, la disciplina, con el cuerpo en tanto individuo, y la biopolítica, como regulación de la población o conjunto de cuerpos, son tecnologías de poder que buscan la gestión y administración de la vida del individuo y de las poblaciones de forma coordinada. Nuestro propósito es tratar de mostrar esta articulación a través de las prácticas y discursos de la educación sexual, y su vínculo estrecho con la escuela como espacio de regulación disciplinaria de saberes y prácticas, en el interior de la cual se producen sujetos. Es en la escuela donde los discursos y prácticas de la sexualidad se normalizan y encauzan con el fin de defender la sociedad en un sentido biopolítico.

\section{La escuela como institución disciplinaria y de control}

Si bien en el pensamiento de Michel Foucault no se plantea un interés directo hacia la educación, es posible vislumbrar entre algunos de sus libros ciertas pistas que nos conducen a saber qué pensaba el filósofo francés al respecto.

En su conferencia de inauguración en el Collège de France en 1970, titulada El orden del discurso, Foucault afirma que existen ciertos procedimientos que permitirán el control de los discursos -prácticas 
discursivas ${ }^{4}$. Entre ellos aparece el ámbito educativo como un lugar donde persiste el acomodamiento social de estos procedimientos, pues para el filósofo: "Todo sistema de educación es una forma política de mantener o de modificar la adecuación de los discursos, con los saberes y los poderes que implican (...)" (2005, p. 44). De esta manera la escuela, como uno de los tantos lugares en los que se puede hablar de educación, hará operar, dar forma, a lo que es posible pensar y decir en el marco de su época. En nuestro caso, la escuela servirá de institución que promoverá ciertos discursos sobre la sexualidad, produciendo un determinado tipo de sujetos sexualizados.

En un primer momento, por ejemplo, el tema de la educación sexual en la escuela estaba implícito en asignaturas como la de biología al plantearse la reproducción de las especies. Posteriormente, la educación sexual parecía exigir su propio espacio debido al aumento de las enfermedades de transmisión sexual, en especial el VIH-SIDA. La educación sexual es tratada, entonces, en términos de prevención, como una cuestión de salud. Actualmente la educación sexual no se preocupa solamente por temas de higiene y salubridad, pues su accionar se ha vuelto fundamentalmente una cuestión moral basada en una relación consigo mismo, donde el eje central será la afectividad (qué tanto me quiero) y la responsabilidad (cuánto me cuido).

La conformación de lo verdadero y lo falso, de lo normal y anormal, incluso de lo pensable e impensable se presenta en el proceso de enseñanza. Allí se llevan a cabo juegos de veridicción que establecerán no solo lo verdadero a partir de su producción, sino también a través de su circulación. Así, los regímenes de verdad que nos subjetivan sirven para otorgar a nuestras prácticas el sentido de legítimas, auténticas, veraces, hasta el momento en el que otros discursos -prácticas discursivas- dicten "su verdad", reconfigurando los principios del pensamiento. Es por esto que de una época a otra la sexualidad deja de ser percibida como un asunto de pecado, para ser pensada en términos médicos y psicoanalíticos, al punto de que en la actualidad se convierte en un

${ }^{4}$ En la obra de Foucault la noción de discurso no hace referencia a un tipo de conocimiento que refleje una realidad, sino a un conjunto de prácticas que al pasar por un juego de veridicción dicen algo en particular. Esa particularidad, esa singularidad, da paso a la constitución de una formación histórica: "es la descripción más precisa, más exacta de una formación histórica en su desnudez", nos dirá Paul Veyne (2015, p. 16).

Traslaviña, K.; Macías, G. (2021). Michel Foucault: escuela, sexualidad y procesos de 
asunto directamente educable y se encuentra enmarcada en una determinada ciencia: la sexología. Cada sociedad construye sus regímenes de verdad, su política general de la verdad y, con base en ello, produce históricamente la normatividad y los mecanismos que permiten aceptar lo verdadero y rechazar lo falso. Veyne sostiene al respecto: “(...) cada uno de estos discursos sucesivos se encuentra implícito en las leyes penales, gestos, instituciones, poderes, costumbres e incluso edificios que los ponen en práctica (...)" (2015, p. 18). Regresando a su conferencia inaugural, Michel Foucault planteará una pregunta que se responde a sí misma, en la cual evoca el tema de la enseñanza: “¿Qué es, después de todo, un sistema de enseñanza, sino una ritualización del habla; sino una cualificación y una fijación de las funciones para los sujetos que hablan; sino la constitución de un grupo doctrinal cuando menos difuso; sino una distribución y una adecuación del discurso con sus poderes y saberes?” (2005, p. 28).

De este modo, para el filósofo francés el tema de la enseñanza presenta características como la ritualización del habla, la cualificación y fijación de funciones, la constitución de un cuerpo doctrinal y la distribución y adecuación del discurso. Así pues, lejos de una mirada que enaltece la educación como un "bien social incuestionable", Foucault planteará cómo la práctica de enseñanza se constituye como "transacciones discursivas" operadas en momentos históricos específicos en busca de la conservación o desplazamiento de determinada verdad, que produce subjetividades. Nos dirá Julio Groppa al respecto: “(...) la enseñanza termina siendo investida como el medio principal por el cual las nuevas generaciones serán sometidas a los juegos de veridicción del presente (...)” (2017, p. 25).

De esta forma, se toma distancia ante la idea de un sujeto soberano, capaz de constituir su propia realidad, para plantear cómo el sujeto es resultado de un conjunto de prácticas que lo subjetivan y constituyen en el marco de un conjunto de saberes y relaciones de poder históricas. ¿Cuál sería el papel de la escuela en este sentido? Desde una analítica foucaulteana, lejos de ser el lugar mediador entre la familia y el paso a la adultez, la escuela es productora de subjetividades, reproductora de discursos y prácticas; es participe de la regulación de ciertos saberes con presunción de verdad. Como espacio disciplinario, la institución escolar se apoyará en ciertos procedimientos que servirán para encauzar el cuerpo de los individuos y la regulación de los saberes. La escuela es una enorme maquinaria orientada a producir cuerpos y verdades; en este caso sobre la sexualidad. 
Con base en su libro de 1975, Vigilary Castigar, se dará cuenta de cómo operaba la escuela en la producción de subjetividades a través del disciplinamiento de los cuerpos, para luego concentrarnos en la reconfiguración de los mecanismos de poder con las sociedades de control.

Para Foucault la disciplina, en términos de poder, tendrá como blanco el cuerpo del individuo. Para ello tendrá como referente discursivo las disciplinas (en el marco del saber) características de las llamadas ciencias humanas. De esta forma, la pedagogía establece un régimen de verdad que tendrá por referencia al sujeto y operará en la constitución de éste. Lejos de ver la pedagogía como una simple mediadora en el desarrollo de los sujetos, se la asume como un saber que opera en la constitución de los individuos. Foucault sostendrá al respecto: "Llamemos 'pedagógica', si quieren, la transmisión de una verdad que tiene la función de dotar a un sujeto cualquiera de aptitudes, capacidades, saberes, etcétera, que no poseía antes y que deberá poseer al termino de esa relación pedagógica (...)" (2014, p. 388). Surge así toda una microfísica del poder, que con sus reglamentos, instrumentos y procedimientos buscan controlar y corregir a los cuerpos, en términos de utilidad económica y docilidad ética, pues "El momento histórico de las disciplinas es el momento en el que nace un arte del cuerpo humano que no tiende únicamente al aumento de sus habilidades, ni tampoco a hacer más pesada su sujeción, sino a la formación de un vínculo que, en el mismo mecanismo, lo hace tanto más obediente cuanto más útil, y viceversa.” (1997, p. 160).

Entre los procedimientos de la disciplina que se buscan recuperar en el presente artículo están la distribución de los individuos en el espacio, el control de su actividad y la organización de la génesis comprendida como economización del tiempo.

Atendiendo al primer procedimiento, la disciplina busca distribuir a los individuos y organizarlos en espacios cerrados. Los espacios de clausura se diversifican, pues ya no se centran en los locos o enfermos solamente. De la misma forma, operan como un tipo de encierro menos perceptible, más discreto, en la formación de sujetos eficaces. Así, por ejemplo, la escuela retoma el modelo del convento para dividirse por zonas y asignar a cada individuo un lugar determinado, y a partir de este lugar poder vigilar su circulación y comunicación. Existe una distribución celular que busca evitar cualquier conglomeración desorganizada de personas, pues la circulación sin 
orden e inutilizable es solucionada por medio de un procedimiento: “(...) para conocer, para dominar y para utilizar. La disciplina organiza un espacio analítico (...)” (2009, p. 166).

Estos lugares determinados en los que se sitúa al individuo deben responder a una utilidad: la dirección de los cuerpos al interior del espacio. El espacio no solo satisface la vigilancia al individuo, sino que también sirve para sacar el mayor provecho a través de su distribución celular. En consecuencia, cada espacio responde a un tipo de clasificación que hace circular a los individuos en un sistema de relaciones. Es por ello que las escuelas se dividen en salones a los que corresponden determinada cantidad de estudiantes. Al interior de los salones de clase cada alumno está clasificado no sólo por su edad, sino por el espacio que ocupa en un pupitre específico. Dentro del salón estará un profesor, quién debe tener el espacio suficiente para observar y vigilar a todos y cada uno de sus alumnos. Cada salón contará con puerta abierta o ventanas grandes, con la suficiente iluminación y ventilación para que el coordinador pueda ejercer una vigilancia general de profesores y alumnos, con fines biopolíticos de control médicos y de disolución de conductas indeseables. Asimismo, la escuela es un lugar más en el que se refuerza la separación entre baños para niños y niñas, para profesores y administrativos, como distinción de sexo y jerarquía. Esta estructura organizacional se reproduce también en hospitales y fábricas.

Cualquier conducta inadecuada como muestras de cariño, caricias "inapropiadas", entrar al baño que no corresponde, no estar en clase, las reuniones grupales sin previa autorización etc., serán corregidas en el marco del encauzamiento de la conducta. Al interior de las escuelas es cada vez menos frecuente el castigo físico como espectáculo ostensible de poder; ahora se busca redireccionar las conductas que se salen del objetivo escolar, y este encauzamiento será apoyado por sesiones terapéuticas que tendrán por base la medicina, la psicología, la psiquiatría y la propia pedagogía. En ese sentido, se hace necesario expandir a otras instituciones (hospital, diván, fábrica) estas formas de proceder ante las conductas anormales, para ofrecer un manejo interno y que éstas no se propaguen a través de la sociedad, pues perjudicaría el bienestar de ésta y la pondría en riesgo en términos biopolíticos.

El segundo procedimiento responde al control de la actividad. Para ello es necesario establecer ritmos, obligar a realizar ocupaciones determinadas y regular ciclos de repetición (p. 175). Los horarios de clase que marcan 
horas exactas de inicio y fin de actividades específicas dentro de un área determinada (de 8am a 9am: Matemáticas, de 9am a 10am: Educación sexual, etc.), son un claro ejemplo de esto. Los programas o planes de estudio definen un esquema cronológico de comportamiento, que va desde lo micro hasta lo macro dentro de la institución escolar. La planeación comienza desde: a) establecer cuáles son las materias más importantes a las que se debe dedicar más tiempo, b) la cantidad de horas por cada materia según su importancia y, c) las temáticas por materia, dependiendo del grado escolar en el que ésta sea enseñada. Así, por ejemplo, la Ley General de Educación de Colombia afirma que "La educación sexual, [debe ser] impartida en cada caso de acuerdo con las necesidades psíquicas, físicas y afectivas de los educandos según su edad" (MEN, 1994). Es una planeación que atraviesa toda la institución escolar, desde los primeros hasta los últimos años: “(...) es un 'programa' que asegura la elaboración del propio acto y controla desde el interior su desarrollo y sus fases, [así] el tiempo penetra el cuerpo y, con él, todos los controles minuciosos del poder (...)" (Foucault, 2009, p. 176).

Dentro de los procesos de enseñanza existe también una relación entre el cuerpo y el gesto, es decir, una actividad específica que el cuerpo logra codificar mediante la repetición. Por ejemplo, la escritura, una postura correcta, la posición de los brazos, las formas de saludar etc., son actividades puestas a repetición y vigilancia. Con la llegada de la Educación sexual a las escuelas, las actividades que se integran a ese tipo de gimnasia tienen que ver con la forma correcta de poner un condón, ejercitarnos en la toma de anticonceptivos diarios o que responden a otras temporalidades igualmente reguladas, la limpieza de los genitales, etc. Si bien estas actividades no se realizan en un espacio público como la escuela, el tema de la enseñanza de éstas en la institución supone su realización en el ámbito privado. Su no realización sería muy evidente a partir de embarazos tempranos, enfermedades de transmisión sexual, infecciones genitales etc. Sobre este punto se refleja una clara articulación entre las tecnologías de poder disciplinarias y biopolíticas, a través de una serie de discursos y prácticas dirigidas a la regulación de las poblaciones.

El tercer procedimiento tiene que ver con la economización del tiempo, para lo cual, muy a la par del control de la actividad, se clasifica a los individuos según el grado escolar y los ejercicios que le convienen en términos de utilidad: 
Es este tiempo disciplinario el que se impone poco a poco frente a la práctica pedagógica, especializando el tiempo de formación y separándolo del tiempo adulto, del tiempo del oficio adquirido; disponiendo diferentes estadios separados los unos de los otros por pruebas graduales; determinando programas que deben desarrollarse cada uno durante una fase determinada y que implican ejercicios de dificultad creciente; calificando a los individuos según la manera en que han recorrido estas series (...) Se conforma así toda una pedagogía analítica, muy minuciosa en su detalle (descompone hasta en sus elementos más simples la materia de enseñanza, jerarquiza en grados exageradamente próximos cada fase del progreso) y muy precoz también en su historia (anticipa ampliamente los análisis genéticos de los ideólogos, de los que aparece como el modelo técnico) (p. 185).

El tiempo tiene un carácter evolutivo, pues su administración a partir de la división busca la utilidad y la totalización de un proceso. Habrá que pensar, por ejemplo, en el tiempo distribuido minuciosamente en los planes de clase. Tiempos regulados con objetivos diseñados en términos de modificación de la conducta y secuenciados entre sí. Ya no es el tiempo global de clase, sino un tiempo segmentado con objetivos, materiales, actividades y formas de evaluación en segmentos cortos. Con el tiempo, la escuela se hace responsable de los cuerpos en términos disciplinarios y biopolíticos.

Ahora bien, si comprendemos que el análisis de Foucault sobre las relaciones entre poder, saber y sujeto son susceptibles de cambio histórico, el estudio que propone el filósofo francés es, entonces, inacabado. En este sentido, en un intento por continuar con los análisis foucaultianos, surge un texto de Gilles Deleuze titulado "post-scriptum sobre las sociedades de control", en donde se plantea la crisis de las sociedades disciplinarias.

Lejos de suponer una superación y sustitución de las sociedades de control sobre las disciplinarias, Deleuze apunta a una transformación y reconfiguración de los mecanismos de poder. Una de las características de las sociedades de control es que se rigen por un régimen de dominación de carácter empresarial. A diferencia de las sociedades disciplinarias que tenían como modelo la fábrica, las sociedades de control tendrán por modelo la empresa. Los procesos de subjetivación se constituyen a partir de la premisa "sé el empresario de ti mismo", "fórmate para un mejor estilo de vida"s. Este

\footnotetext{
5 Byung-Chul Han brinda una mirada más profunda a lo que actualmente se denomina "sujeto del rendimiento", quien tiene por base la autoexplotación para una supuesta realización de sí mismo (Han, 2012).
} 
sujeto, empresario de sí mismo, tiene que actualizarse para poder cumplir con los requisitos que el mercado exige, y ese actualizarse requiere incluso que sea fuera de las instituciones de encierro como la escuela, lo que afecta a la educación institucionalizada, que empieza a regirse también bajo esta lógica empresarial. Deleuze afirma entonces que "El principio modular del 'salario al mérito' no ha dejado de tentar a la propia educación nacional: en efecto, así como la empresa reemplaza a la fábrica, la formación permanente tiende a reemplazar a la escuela, y la evaluación continua al examen. Lo cual constituye el medio más seguro para librar la escuela a la empresa" (1991, p. 2).

Otra de las características será la forma en la que opera el control sobre los sujetos. Las nuevas tecnologías de la información y comunicación permitieron la reconfiguración de los mecanismos de poder. La vigilancia y el control operan a través de dispositivos más flexibles y menos perceptibles, lo cual extiende su campo de acción y permite utilizarlos incluso fuera de las instituciones de encierro. En términos biopolíticos, la ayuda de dispositivos mucho más flexibles y cambiantes (cámaras, redes sociales, publicidad, celulares), que permiten modular y dirigir las conductas de los sujetos a partir del control, se constituye como mecanismos de gobierno más eficaces, que permiten inducir y direccionar cierta forma de vida, ahora bajo la lógica empresarial.

La lógica empresarial, con sus prácticas y discursos, afecta directamente las instituciones educativas. Éstas se ven obligadas a adoptar las formas administrativas de la empresa y un discurso ético que propone como ejes rectores del accionar la búsqueda de calidad y la excelencia (Anzaldúa, 2017, p. 65). Si en las sociedades disciplinarias los procesos de subjetivación eran comunes en la fábrica y la escuela, ahora, a raíz de los desarrollos del modelo económico capitalista, el vínculo es entre la empresa y las instituciones educativas. De esta forma, los planes de estudios y las pedagogías imperantes buscan responder a las dinámicas empresariales.

\section{Escuela y procesos de subjetivación}

Anteriormente mencionamos que el sujeto es el resultado de un proceso histórico. Al respecto, Foucault afirma: "Hay dos significados de la palabra sujeto: sometido a otro a través del control y la dependencia, y sujeto atado a su propia identidad por la conciencia o el conocimiento de sí mismo. Ambos 
significados sugieren una forma de poder que subyuga y somete" (1988, p. 7). En consecuencia, el individuo interioriza los discursos catalogados como verdaderos, que marcan la realidad en la que vive y se subjetiva a partir de ellos. De esta forma se dictará a sí mismo unas prácticas adecuadas de vivir la sexualidad, según la escuela, y la necesidad de educarse bajo ese deber ser.

Los procesos de subjetivación imperantes en nuestra época, producidos por instituciones como las escolares, y dados en el marco de las sociedades de control, están basados en la autorregulación. El sujeto deber ser "responsable" de sí mismo, rigiéndose por la lógica de los discursos y prácticas que la misma escuela se encarga de reproducir, controlar o transformar. Esto significa que un estudiante útil y dócil atiende a los discursos de la sexualidad moderna, basados principalmente en la medicina y la psicología, cuando usa anticonceptivos, tiene buena higiene, quiere su cuerpo, tiene cuidado de con quién se relaciona y piensa en un proyecto de vida de calidad. A su vez, se adopta un discurso moral en torno a la sexualidad, el cual sigue el ejemplo del cristianismo y la Antigüedad, al tener como imperativo "esperar el momento indicado", pero ya no con los mismos fines. En el cristianismo, esperar el momento indicado tenía que ver con una reproducción sana del hombre que fuera semejanza de $\operatorname{Dios}^{6}$, y en la Antigüedad hacía referencia al autogobierno de los placeres como muestra de una moderación adecuada para no afectar el cuerpo ${ }^{7}$. Sin embargo, esperar el momento indicado tiene que ver ahora con una serie de metas que darán cuenta de una sustentabilidad económica. Esto significa "preocúpate primero por terminar la escuela, luego la universidad, después por encontrar trabajo y después piensas en eso". Esto no supone que las prácticas sexuales se pospongan, sino solamente que se debe imponer un buen uso del tiempo, lo que tiene que ver con un proyecto de vida organizado.

\footnotetext{
${ }^{6}$ Cuestión de la que se ocupa Foucault en el segundo tomo de Historia de la sexualidad, subtitulado Uso de los placeres. En este escrito se ahondará en las prácticas referentes a la sexualidad enmarcadas en el cristianismo. Estas prácticas se argumentan bajo una verdad fundada en la palabra de Dios, para quien hay que esperar el momento oportuno del acto sexual y la reproducción del hombre a su imagen y semejanza.

7 Tema del cual se ocupa profundamente Foucault en el tercer tomo de Historia de la sexualidad, subtitulado Inquietud de sí, en el que el filósofo francés ahondará en las prácticas del cuidado de sí que se ejercían en la antigua Roma. Dichas prácticas responden a una verdad fundada en el gobierno del sí mismo para una estilización de la vida como obra de arte, y ya no tomarán en cuenta solamente el uso de los placeres. En este punto, Foucault argumenta que la sexualidad no es el único aspecto que gira alrededor de la constitución del sujeto como sujeto ético.
} 
En este sentido, es necesario que los sujetos se encuentren realizando actividades dentro y fuera de la escuela, pues esta formación permanente posibilitaría el aprendizaje de competencias requeridas por el modelo económico. Estas actividades son incentivadas por el sistema educativo a partir de un discurso del emprendimiento, donde el rol del maestro parece ser el de promotor de autoaprendizaje mediante el coaching. Esto tiene que ver también con el manejo del tiempo libre, pues según el Ministerio de Educación Nacional de Colombia (MEN), por ejemplo, uno de los "factores intrapersonales" según los "Determinantes Sociales del embarazo en la adolescencia" es el manejo del tiempo libre (2014, p. 17). Este discurso de prevención va anudado a prácticas discursivas que ponen de relieve el tema de la responsabilidad sexual, la que en términos biopolíticos evita una masificación de personas enfermas por transmisiones sexuales, infecciones o embarazos a temprana edad.

Actualmente, los discursos acerca de la sexualidad en las escuelas otorgan también gran importancia a los valores. Es por ello que el amor resulta ser uno de los principales pilares de las escuelas. Amar en primera instancia tu propio cuerpo te permitirá amar el de los otros, y será a partir de este vínculo amoroso que se puede llevar a cabo, y con total responsabilidad, la consumación del acto.

Lejos de querer hacer juicios de valor sobre el modelo educativo que tiene correlato con un modelo económico, y que se transmite a partir del Estado como intermediario, lo que se ha pretendido mostrar es que la escuela, al utilizar el saber de la sexualidad, no debe comprenderse como una cuestión de progreso o liberación, sino como una transfiguración de los mecanismos de poder, sin que esto signifique suponer que sean buenos o malos.

\section{Conclusiones}

La escuela, como institución disciplinaria, produce subjetividades. La educación escolar será el espacio de institucionalización y normalización de las prácticas destinadas a establecer una forma de vivir la sexualidad mediante su educación. La ciencia de la sexualidad, como un saber científico y legítimo, debe ser público, conocido, divulgado dentro de instituciones como la escuela y la familia. La escuela funciona como el espacio para construir una sexualidad que pueda ser administrada a través de la configuración de prácticas y saberes normalizados, reglamentando formas específicas y 
moralmente aceptadas para un deber ser de la sana sexualidad. La vida sexual se convierte en objeto de vigilancia, administración, control y regulación, no de prohibición. Tal como menciona Foucault en Historia de la sexualidad I. La voluntad de saber o en su curso Los anormales, la característica principal de regulación de los saberes sobre la sexualidad no es el modelo de la represión o la censura, sino el de la proliferación de los discursos. La escuela es uno de los espacios donde el saber sobre la sexualidad circula en articulación con todo un conjunto de prácticas de control de los espacios, los gestos y el tiempo, y se convierte en objeto de educación. En la articulación entre disciplina y biopolítica, la sexualidad debe educarse como parte de la constitución de un sujeto sexualizado. Por ejemplo, uno de los planteamientos del Programa de Educación para la Sexualidad y Construcción de Ciudadanía (PESCC), diseñado por el Ministerio de Educación Nacional de Colombia, sostiene:

Educar la sexualidad es mucho más que transmitir conocimientos e información acerca de la sexualidad y la reproducción. No basta con enseñar las características biológicas de hombres y mujeres, o los métodos para prevenir un embarazo. Educar para la sexualidad es precisamente brindar herramientas conceptuales, actitudinales, comunicativas y valorativas que permitan a los adolescentes tomar decisiones con relación a su sexualidad que se correspondan con lo que quieren, sueñan y esperan de su realidad (2008).

En consecuencia, la escuela funciona como el espacio para dirigir conductas y formar sujetos, mediante un saber como lo es la sexualidad. Una sexualidad que pueda ser administrada a través de la configuración de prácticas (lo actitudinal) y saberes (herramientas conceptuales) normalizados, y que, a su vez, reglamenta formas determinadas, y moralmente admitidas, para un deber ser como sujeto sexualizado.

Con ayuda de los saberes pedagógico, psiquiátrico, psicológico, psicoanalítico, médico y pedagógico, se instauran unas formas determinadas de vivir la sexualidad, ya que estos saberes forman parte de los tipos de racionalidades que surgen alrededor de ésta. Cualquier tipo de anomalía o patología que ponga en peligro la estabilidad y bienestar de la población, será encauzada de inmediato bajo algún discurso científico. Actualmente, organismos internacionales como la UNESCO dan cuenta de las iniciativas relevantes para el derecho humano a la educación sexual integral, ya que "reconoce que la ESI (Educación Sexual Integral) debe comenzar en la escuela primaria y 
continuar en todos los niveles formales y no formales de la educación" (2014, p. 16), para lo cual, contará con el apoyo de organizaciones internacionales como la OMS:

Para la Organización Mundial de la Salud (OMS), es fundamental que la ESI se inicie en los primeros años de la educación básica. La OMS también ha proporcionado orientación específica sobre cómo la educación sexual debería ser incorporada en los programas escolares y recomienda que la ESI se enseñe como una materia independiente, en lugar de incorporarse a otras asignaturas (p. 16).

En suma, escuela y sexualidad se anudan para subjetivar individuos en el marco de un discurso responsable, logrando así que los sujetos mismos estén dispuestos a tomar las riendas de su propio proyecto de vida, pues tal como afirma el MEN: "Una educación de calidad es aquella que forma mejores seres humanos, ciudadanos con valores éticos, respetuosos de lo público, que ejercen los derechos humanos y conviven en paz (...), una educación que genera oportunidades legítimas de progreso y prosperidad para ellos y para el país" (2014, p. 24), lo cual, en términos disciplinarios se interpreta como un individuo responsable de su cuerpo y, en términos biopolíticos, como una sociedad con una forma de vida sexualmente sana y educada.

\section{Referencias}

Anzaldúa, R. (2017). La universidad en las sociedades de control. M. Jiménez y A. Valle Vázquez (Eds.), Sociología y pedagogía, Defender la universidad (pp. 65-83). México: UNAM-FES Acatlán.

Castro, E. (2011). Diccionario Foucault, Temas, conceptos y autores. Buenos Aires: Siglo XXI.

Deleuze, G. (1991). Posdata sobre las sociedades de control. C. Ferrer (Ed.), El lenguaje literario. Montevideo: Editorial Nordan. http://www. fundacion.uocra.org/documentos/recursos/articulos/Posdata-sobre-lassociedades-de-control.pdf

Foucault, M. (1988). El sujeto y el poder. Revista Mexicana de Sociología, 50 (3). pp. 3-20. https://doi.org/10.2307/3540551

Foucault, M. (1997). Historia de la sexualidad I La voluntad de saber. Madrid: Siglo XXI. 
Foucault, M. (2000). Defender la sociedad. Buenos Aires: Fondo de Cultura Económica.

Foucault, M. (2005). El orden del discurso. Buenos Aires: Tusquets.

Foucault, M. (2009). Vigilar y Castigar. Nacimiento de la prisión. México: Siglo XXI.

Foucault, M. (2014). Hermenéutica del sujeto. México: Fondo de Cultura Económica.

Groppa, J. (2017). La teorización foucaultiana y sus efectos sobre el campo educativo. O. Pulido, M. Suárez, y O. Espinel (Compiladores), Pensar de otro modo. Herramientas filosóficas para investigar en educación (pp. 17-28). Tunja: Universidad Pedagógica y Tecnológica de Colombia.

Han, B. (2012). La sociedad del cansancio. Barcelona: Herder.

Ministerio de Educación de Colombia (1994). Ley 115 de febrero 8 de 1994. https://www.mineducacion.gov.co/1621/articles-85906_archivo_pdf. pdf

Ministerio de Educación Nacional de Colombia (2008). Educación para la sexualidad: derecho de adolescentes y jóvenes, y condición para su desarrollo. Revista Altablero, 47. https://www.mineducacion.gov. co/1621/article-173947.html

Ministerio de Educación Nacional de Colombia (2014). Programa Educación para la Sexualidad y Construcción de Ciudadanía. https:// www.mineducacion.gov.co/1621/articles-171578_doc_modulo1. doc\#: : :text=El\%20Programa $\% 20 \mathrm{de} \% 20$ Educaci $\% \mathrm{C} 3 \%$ B3n $\% 20$ para,sostenibilidad\%20de\%20una\%20pol\%C3\%ADtica\%20de

UNESCO (2014). Educación Integral de la Sexualidad: Conceptos, enfoques $y \quad$ competencias. http://unesdoc.unesco.org/ images/0023/002328/232800S.pdf

Veyne, P. (1984). Cómo se escribe la historia. Foucault revoluciona la historia. Madrid: Alianza.

Veyne, P. (2015). Foucault, pensamiento y vida. México: Paidós. 